Aufsatz ÖR

Prof. Dr. Wolfgang Kahl, M. A.* und Dr. James Bews

\title{
Die Wiedereinsetzung in den vorigen Stand im öffentlichen Recht - Teil 2
}

https://doi.org/10.1515/jura-2018-0074

Der Beitrag behandelt - im Anschluss an Teil 1 (JURA 2018, Heft 3) - die Begründetheitsvoraussetzungen eines Antrags auf Wiedereinsetzung in den vorigen Stand im öffentlichen Recht und geht daneben auf Besonderheiten der Wiedereinsetzung im Europarecht sowie auf Rechtsschutzfragen ein.

\section{Begründetheit}

Dem Antrag auf Wiedereinsetzung in den vorigen Stand ist stattzugeben, wenn der Antragsteller glaubhaft macht, dass er das die Fristversäumnis begründende Hindernis nicht verschuldet hat. Erforderlich ist somit dreierlei: (a) Das Hindernis ist nicht durch den Antragsteller verschuldet, (b) das Fristversäumnis beruht kausal auf diesem Hindernis und (c) der Antragsteller macht dies glaubhaft.

Klausurtipp: In der Klausur bietet sich regelmäßig eine einheitliche Prüfung der Punkte a) und c) an. Auf die Frage der Kausalität ist nur einzugehen, wenn Anhaltspunkte dafür vorliegen, dass diese problematisch ist.

\section{a) Unverschuldetes Hindernis}

Dem Fristversäumnis muss ein von dem Antragsteller nicht verschuldetes ${ }^{1}$ Hindernis zugrunde liegen. Dabei liegt ein

1 Bei einem verschuldeten Fristversäumnis handelt es sich strenggenommen um eine Obliegenheitsverletzung; s. Maunz/SchmidtBleibtreu/Bethge/Hömig BVerfGG, 52. EL. 2017, § 93 Rn. 46.

*Kontaktperson: Wolfgang Kahl, ist Inhaber des Lehrstuhls für Öffentliches Recht, insbesondere deutsches und europäisches Verwaltungsrecht, an der Universität Heidelberg.

James Bews, ist Richter auf Probe am Verwaltungsgericht Berlin; der Beitrag spiegelt lediglich seine persönliche Meinung wider.
Verschulden vor, wenn der Antragsteller das Hindernis vorsätzlich ${ }^{2}$ oder fahrlässig verursacht hat. ${ }^{3}$ Die Fahrlässigkeit bestimmt sich dabei nicht nach einem objektiven Maßstab. ${ }^{4}$ Zum Schutz von Art. 19 IV 1, 103 I GG ist vielmehr die dem individuell Betroffenen subjektiv zumutbare Sorgfalt maßgeblich. ${ }^{5}$ Diese wird freilich insofern objektiviert, als eine typisierende Betrachtung angestellt wird. ${ }^{6}$ Nach diesem gemäßigt subjektiven Maßstab handelt der Antragsteller fahrlässig, wenn er diejenige Sorgfalt außer Acht lässt, »die für einen gewissenhaft und sachgemäß Prozessführenden geboten ist und ihm nach den gesamten Umständen zuzumuten war « ${ }^{7}$. Dem Wiedereinsetzungsantrag muss sich entnehmen lassen, dass die Fristversäumung auch bei Anwendung der entsprechenden Sorgfalt nicht hätte vermieden werden können. ${ }^{8}$ Neben dem Eigenverschulden des

2 Vorsätzliche Fristversäumnisse kommen praktisch nicht vor; s. Posser/Wolff/Brink BeckOK VwGO, 43. Ed. 2017, § 60 Rn. 7. Für Beispiele s. aber Kummer Wiedereinsetzung in den vorigen Stand, 2003, Rn. $247 \mathrm{ff}$.

3 Dieser Verschuldensmaßstab wird zumeist direkt aus § 60 VwGO (bzw. den entsprechenden Vorschriften) abgleitet, ohne Bezugnahme insbesondere auf § 276 BGB, vgl. nur Sodan/Ziekow/Czybulka VwGO, 4. Aufl. 2014, § 60 Rn. 42; Gärditz/Krausnick VwGO, 2013, § 60 Rn. 19f. Es ist aber auch vertretbar, § 276 BGB analog heranzuziehen. 4 So aber Burkiczak/Dollinger/Schorkopf/Hammer BVerfGG, 2015, § 93 Rn. 41; Kuhla/Hüttenbrink Der Verwaltungsprozess, 3. Aufl. 2002, Rn. 405; Mann/Sennekamp/Uechtritz/Mattes VwVfG, 2014, § 32 Rn. 25; Ziekow VwVfG, 3. Aufl. 2013, § 32 Rn. 7.

5 Schoch/Schneider/Bier/Steinbeiß-Winkelmann VwGO, 33. EL. 2017, $\S 60$ Rn. 19; Posser/Wolff/Brink BeckOK VwGO, 43. Ed. 2017, § 60 Rn. 8; Kopp/Ramsauer VwVfG, 18. Aufl. 2017, § 32 Rn. 20 a; Müller NJW 1993, 681, 681.

6 BVerfGE 86, 280, 284f.; BVerwGE 50, 248, 254; Bader u.a./v. Albedyll VwGO, 6. Aufl. 2014, § 60 Rn. 2; Sodan/Ziekow/Czybulka VwGO, 4. Aufl. 2014, § 60 Rn. 42; Gärditz/Krausnick VwGO, 2013, § 60 Rn. 19; Eyermann/Schmidt VwGO, 14. Aufl. 2014, § 60 Rn. 6; Fehling/Kastner/Störmer/Schwarz Verwaltungsrecht, 4. Aufl. 2016, § 32 VwVfG Rn. 18.

7 Redeker/v. Oertzen VwGO, 16. Aufl. 2014, § 60 Rn. 3; ähnlich OVG Bautzen NVwZ-RR 2017, 755; Kopp/Schenke VwGO, 23. Aufl. 2017, $\S 60$ Rn. 9.

8 BVerwG, Beschl. v. 6.12.2000, Buchholz 310 § 60 VwGO Nr. 236; OVG Bautzen NVwZ-RR 2017, 755. 
Antragstellers (аa.) kann ihm dabei auch Fremdverschulden zugerechnet werden (bb.).

\section{aa) Eigenverschulden}

Fall 2 A hat gegen die seinem Nachbarn N erteilte Baugenehmigung Widerspruch eingelegt, der zurückgewiesen wurde. Der mittellose A möchte nun Klage vor dem Verwaltungsgericht erheben, verfügt aber nicht über die erforderlichen finanziellen Mittel. Daher stellt er einen Antrag auf Prozesskostenhilfe (PKH), der nach acht Wochen wegen mangelnder Erfolgsaussichten zurückgewiesen wird. Kann A gegen die nunmehr in Bestandskraft erwachsene Baugenehmigung gerichtlich vorgehen?

Maßgeblich ist grundsätzlich, ob der Antragsteller das Fristversäumnis selbst verschuldet hat. Diesbezüglich haben sich in der Rechtsprechung bestimmte Fallgruppen herausgebildet, wobei zwischen Umständen, die in der Person des Antragstellers begründet sind (1), und sonstigen Umständen (2) unterschieden wird.

(1) Befand sich der Antragsteller zum Zeitpunkt des fristauslösenden Ereignisses im Urlaub, ist ein Verschulden beim Fehlen besonderer Vorkehrungen nur dann anzunehmen, wenn der Urlaub entweder länger als sechs Wochen dauerte ${ }^{9}$ oder der Antragsteller mit einer baldigen Zustellung rechnen musste. ${ }^{10}$ Ausländer müssen Fristversäumnisse aufgrund von Sprachschwierigkeiten vermeiden, indem sie sich in angemessener Frist Gewissheit über den Inhalt eines Schriftstücks verschaffen. ${ }^{11}$ Daneben ist dem Antragsteller eine Berufung auf seine Unkenntnis der Rechtslage verwehrt. ${ }^{12}$

Ist der Antragsteller aufgrund von Krankheit ${ }^{13}$ oder einer akuten privaten Krisensituation ${ }^{14}$ an der fristgemäßen Einlegung des Rechtsbehelfs gehindert, kommt eine Wiedereinsetzung in Betracht.

Hat er einen PKH-Antrag gestellt, über den vor Fristablauf noch nicht entschieden wurde, gilt Folgendes:

9 BVerfGE 41, 332, 335f.; BVerwGE 77, 157, 161.

10 BVerwG NJW 1975, 1574, 1575. Den Geschäftsführer einer GmbH treffen auch bei kurzfristiger Urlaubsabwesenheit Pflichten zur Einrichtung einer Vertretung; s. VGH Mannheim VBlBW 2016, 328, 329.

11 BVerfGE 86, 280, 285; BVerwG DVBl. 1978, 888; VGH München NJW 1997, 1324, 1325. Anders für das Strafverfahren BVerfGE 42, 120, $123 \mathrm{ff}$.

12 BVerwG NVwZ-RR 1999, 538, 538f.; VGH Mannheim VBlBW 1991, 215. Auch ein Irrtum über die Erfolgsaussichten begründet keinen Wiedereinsetzungsgrund; s. OVG Lüneburg NVwZ-RR 2008, 356.

13 BVerwG Beschl. v. 22.7.2008 - 5 B 50/08, Rn. 7 (juris). Die Krankheit muss jedoch so schwer wiegen, dass er außer Stande ist, einen Bevollmächtigten zu beauftragen.

14 Sodan/Ziekow/Czybulka VwGO, 4. Aufl. 2014, §60 Rn. 76; MünchKomm-ZPO/Stackmann, 5. Aufl. 2016, § 233 Rn. 55.
Dem Antragsteller darf seine Mittellosigkeit nicht angelastet werden, sodass das Fristversäumnis als unverschuldet anzusehen ist. ${ }^{15}$ Grundsätzlich unbeachtlich ist, ob ihm die beantragte PKH auch zugesprochen wird. Ein Verschulden ist dagegen anzunehmen, wenn sein $\mathrm{PKH}-$ Antrag formal aufgrund der unvollständigen Einreichung der Unterlagen abgewiesen wurde ${ }^{16}$ oder offensichtlich erfolglos war. ${ }^{17}$ Bei einem gemäß $§ 188$ S. 2 VwGO gerichtskostenfreien Verfahren ist die Sachlage anders. Hier liegt kein Wiedereinsetzungsgrund vor, weil der Antragsteller im Falle eines Unterliegens keine Gerichtskosten befürchten muss. ${ }^{18}$

(2) Als sonstige (nicht in der Person des Antragstellers liegende) Umstände sind folgende Fallgruppen anerkannt:

Beruht das Fristversäumnis auf einer fehlenden ${ }^{19} \mathrm{Be}-$ gründung oder Anhörung, ist gemäß § 45 III (L)VwVfG ein unverschuldetes Hindernis anzunehmen.

Bei Verzögerungen in der Übermittlung eines Schreibens kann der Antragsteller grundsätzlich davon ausgehen, dass ein Postanbieter ${ }^{20}$ innerhalb der Bundesrepublik eine Übermittlung innerhalb von drei Werktagen gewährleistet. ${ }^{21}$ Selbst wenn - z. B. wegen eines bevorstehenden Feiertags - mit einem erhöhten Postaufkommen $\mathrm{zu}$ rechnen ist, kann der Antragsteller auf eine ordnungsgemäße Zustellung vertrauen. ${ }^{22}$ Eine Ausnahme gilt allenfalls bei außergewöhnlichen Ereignissen wie einem angekündigten Poststreik. ${ }^{23}$ Bei dem Einsatz anderer $\mathrm{Zu}$ stelldienste hat der Antragsteller zu prüfen, ob diese Gewähr für eine rechtzeitige Übermittlung bieten. ${ }^{24}$ Der Antragsteller muss das Schriftstück zutreffend adressiert

15 BVerfG NVwZ 2003, 341, 341f.; BVerwG NVwZ 2002, 992, 992; NVwZ 2004, 111.

16 Hierzu sowie zu Ausnahmen von diesem Erfordernis VGH Mannheim VBlBW 2017, 256, 257.

17 Meyer NJW 1995, 2139, 2140.

18 BVerwG NVwZ-RR 1989, 665, 666; Strnischa NVwZ 2005, 267, $269 \mathrm{f}$.

19 Nicht erfasst wird dagegen eine fehlerhafte, d.h. materiell unrichtige Begründung; vgl. Stelkens/Bonk/Sachs VwVfG, 9. Aufl. 2018, $\S 45$ Rn. 155.

20 Dies gilt nicht nur für die Deutsche Post, sondern für sämtliche Postanbieter, die der Universaldienstverpflichtung nach §§ $11 \mathrm{ff}$. PostG i.V.m. PUDLV unterliegen; s. Gärditz/Krausnick VwGO, 2013, $\S 60$ Rn. 41.

21 BVerfG NJW 1994, 244, 245.

22 BVerfGE 41, 341, 344; 42, 258, 260; 54, 80, 84; NJW 1994, 244, 245; VGH Mannheim NJW 1996, 2882, 2883.

23 BVerfG NJW 1995, 1210, 1211. In diese Richtung bereits BVerfGE 50, 1, 4.

24 OVG Münster NVwZ-RR 2007, 141, 141f. Beim Kurierdienst des Anwaltsvereins ist dies anzunehmen; s. BVerfG NJW 2000, 2657, 2658. 
haben. ${ }^{25}$ Eine unzureichende Frankierung ist dagegen unschädlich, wenn das Schriftstück fristgerecht eingeht und das Gericht die Annahme verweigert. ${ }^{26}$ Beim Einsatz eines Telefax trifft den Antragsteller die Pflicht, die ordnungsgemäße Absendung anhand des Sendeberichts zu kontrollieren. ${ }^{27}$ Für Übersendungsfehler, die in der Risikosphäre des Empfängers liegen (z. B. Defekt des gerichtlichen Geräts), trägt er keine Verantwortung. ${ }^{28}$

Diese Grundsätze gelten unabhängig von dem Zeitpunkt der Übermittlung. Der Antragsteller hat das Recht, die gesetzliche Frist auszunutzen. ${ }^{29}$ Teilweise ist aber die Rede davon, dass den eine Frist ausnutzenden Antragsteller erhöhte Sorgfaltspflichten treffen..$^{30}$ Dies ist aber lediglich so zu verstehen, dass er einen Übermittlungsweg wählen muss, der den rechtzeitigen Eingang gewährleistet. ${ }^{31}$ Versendet der Antragsteller drei Tage vor Fristablauf ein Übergabeeinschreiben, sind ihm Verzögerungen zuzurechnen, die sich daraus ergeben, dass der Adressat von dem Postzusteller nicht angetroffen wurde. ${ }^{32}$ Er kann deshalb nicht davon ausgehen, dass der Empfänger am letzten Tag der einzuhaltenden Frist das Einschreiben erhalten wird. Die Versendung eines Telefax hat der Antragsteller so rechtzeitig zu beginnen, dass unter gewöhnlichen Umständen mit dem Abschluss der Übertragung noch am Tag des Fristablaufs zu rechnen ist. ${ }^{33}$ Die Rechtsprechung legt hier einen strengen Maßstab an und fordert, dass der Antragsteller in den Abendstunden einen Sicherheitszuschlag von 20 Minuten berechnet, um dem Umstand Rechnung zu tragen, dass das gerichtliche Faxgerät kurz vor Mitternacht regelmäßig in besonderem Maße belegt ist. ${ }^{34}$

Lösung Fall 2 A kann eine Anfechtungsklage gegen die Baugenehmigung in Gestalt des Widerspruchsbescheids (vgl. \& 79 I Nr. 1

25 BVerwGE 55, 61, 64. Nach BVerfG NJW 2001, 1566, 1567 soll eine unvollständige Adressierung dagegen unschädlich sein. Dazu auch Kummer Wiedereinsetzung in den vorigen Stand, 2003, Rn. 297.

26 BVerwG ZLA 1975, 127; OVG Weimar Urt. v. 28.4.2005 - 4 KO 393/08, Rn. 98 (juris). Zweifelnd dagegen OVG Hamburg NJW 1995, 3137, 3138.

27 BVerwG NJW 2008, 932, 932f.

28 BVerfG NJW 1996, 2857, 2857 f.; VGH Mannheim NJW 1994, 538.

29 BVerfGE 69, 381, 385; VGH München NVwZ-RR 2005, 4, 5.

30 BVerwG DÖV 1981, 180; NJW 1985, 1178; Eyermann/Schmidt VwGO, 14. Aufl. 2014, § 60 Rn. 8.

31 BVerwG DVBl. 1966, 692f.; Schoch/Schneider/Bier/SteinbeißWinkelmann VwGO, 33. EL. 2017, § 60 Rn. 40.

32 OVG Münster NJW 1987, 1353, $1353 \mathrm{f}$.

33 BVerfG NJW 2000, 574; VGH München NJW 2014, 2459, 2459.

34 BVerfGE 135, 126 Rn. 36; BVerwG NVwZ-RR 2015, 392, 392f. Zur Belegung des Telefaxgeräts des BVerfG s. BVerfG NJW 2006, 1505, 1506. Krit. Lenz/Hansel BVerfGG, 2. Aufl. 2015, § 93 Rn. 63 f.
VwGO) erheben. Zwar hat er die Einmonatsfrist des $\S 74$ I VwGO versäumt. Ein Wiedereinsetzungsantrag hätte aber Aussicht auf Erfolg, da das Fristversäumnis auf der Nichtbescheidung des PKHAntrags beruht und somit als unverschuldet anzusehen ist. Unbeachtlich ist insofern, dass der PKH-Antrag seinerseits nicht erfolgreich war.

\section{bb) Verschuldenszurechnung}

Fall 3 Vertreten durch seinen Rechtsanwalt $R$ legt $K$ eine Woche nach Zustellung eines Urteils des OVG Revision gegen dieses ein. $R$ beauftragt seinen Stationsreferendar S, die Frist für die Revisionsbegründung zu berechnen und ihm eine Woche vor Ablauf dieser Frist einen Entwurf für die Begründungsschrift vorzulegen. Nach Rücksprache mit seiner Lerngruppe geht $S$ davon aus, dass die Revision zwei Monate nach ihrer Einlegung zu begründen ist. Er legt dem R daher zwei Monate nach Zustellung des Urteils seinen Entwurf vor, den $R$ am Folgetag beim BVerwG einreicht. Das $B V e r w G$ verwirft die Revision mangels fristgemäßer Begründung als unzulässig. Hat ein Antrag des K auf Wiedereinsetzung in die Revisionsbegründungsfrist Aussicht auf Erfolg?

Dem Antragsteller ist auch das Verschulden seines gesetzlichen Vertreters sowie seines Prozessbevollmächtigten und sonstiger rechtsgeschäftlicher Vertreter zuzurechnen (§ 173 S. 1 VwGO i. V.m. § 51 II bzw. § 85 ZPO). ${ }^{35}$ Diese Vorschriften sind verfassungsrechtlich nicht $\mathrm{zu}$ beanstanden und finden auch in Verfahren Anwendung, die durch eine besondere Grundrechtsrelevanz geprägt sind. ${ }^{36}$

In Ermangelung einer mit $\S 278$ BGB vergleichbaren Vorschrift ist eine Zurechnung des Verschuldens sonstiger Hilfspersonen (z. B. Bote) dagegen nicht möglich. ${ }^{37}$ Im Einzelfall kann die Abgrenzung solcher Hilfspersonen von den rechtsgeschäftlich Bevollmächtigten aber schwierig sein. So ist bei einer Mandatierung einer Kanzlei das Verschulden eines Sozius grundsätzlich zurechenbar, ${ }^{38}$ während ein angestellter Anwalt ${ }^{39}$ oder ein sonstiger juristischer Mitarbeiter ${ }^{40}$ nur dann als rechtsgeschäftlich bevollmächtigt gilt, wenn er eine hinreichend selbstständige Stellung hatte.

Selbst wenn das Fristversäumnis aber auf dem Verschulden einer Hilfsperson beruht, kann dies dem Antragsteller zugerechnet werden, wenn der von ihm beauftragte

35 Vgl. auch §§ 32 I 2 VwVfG; 93 II 6 BVerfGG, 73 VI 6, 155 FGO; 202 SGG.

36 Für das Asylverfahren vgl. BVerfGE 60, 253, 267 ff. Im Kontext der Kriegsdienstverweigerung BVerwGE 49, 252, 257 ff.; NVwZ 1985, 34, $34 \mathrm{f}$.

37 BVerwG NJW 1992, 63, 64.

38 Müller NJW 1993, 681, 684 m.w. N.

39 BVerwG NJW 1985, 1178; NVwZ 2004, 1007, 1008.

40 Gärditz/Krausnick VwGO, 2013, § 60 Rn. 49. 
Bevollmächtigte seinerseits hinsichtlich des Handelns der eingesetzten Hilfsperson fahrlässig handelte. Der Bevollmächtigte muss nachweisen, dass die eingesetzte Hilfsperson sorgfältig ausgewählt sowie gut ausgebildet, erprobt und überwacht wurde. ${ }^{41}$ Die Übertragung der Übermittlung eines fristwahrenden Schriftstücks an einen unzuverlässigen Büroangestellten ist dem Antragsteller als Verschulden seines Rechtsanwalts zurechenbar. ${ }^{42}$ Zudem dürfen bestimmte Aufgaben nicht auf das Büropersonal übertragen werden, sondern sind von dem Rechtsanwalt selbst zu bearbeiten. Dies gilt regelmäßig ${ }^{43}$ für komplexe Fristberechnungen wie die Revisionsbegründungsfrist ${ }^{44}$ oder die Berufungsbegründungsfrist ${ }^{45}$. Auch eine Übertragung auf einen Stationsreferendar ist in diesen Fällen unzulässig. ${ }^{46}$ Ein Rechtsanwalt darf sich auch eines nicht bei ihm angestellten Boten bedienen, wenn ihm dieser persönlich bekannt ist, er entsprechend unterrichtet wurde und sich bereits mehrfach zuvor in ähnlichen Fällen als zuverlässig erwiesen hat; zudem muss der Bote auf den drohenden Fristablauf und die Notwendigkeit der Fristwahrung ausdrücklich hingewiesen worden sein. ${ }^{47}$

Neben der Auswahl und Überwachung seiner Hilfspersonen treffen den Rechtsanwalt noch weitere, erhöhte Sorgfaltsanforderungen. Er muss auch bei einer kurzfristigen Abwesenheit Anweisungen treffen, um die Einhaltung von Fristen und Terminen zu gewährleisten. ${ }^{48}$ Im Gegensatz zu seinem Mandanten kann er also nicht ohne besondere Vorkehrungen in den Urlaub fahren. Zudem hat er organisatorische Vorkehrungen zur Gewährleistung eines ordnungsgemäßen Geschäftsablaufs in Gestalt von Eingangs-, Ausgangs- und Unterschriftenkontrollen zu treffen. ${ }^{49}$

Die Verschuldenszurechnung bei Behörden verläuft grundsätzlich parallel. Hat die Behörde einen Vertreter mit Befähigung zum Richteramt, gilt § 173 S. 1 VwGO i.V.m.

41 Vgl. nur BVerwGE 27, 36, 37.

42 BGH NJW 1988, 2045, 2045. Vgl. auch OVG Bautzen NVwZ-RR 2017, 755, $755 \mathrm{f}$.

43 Eine Ausnahme gilt in Fällen, in denen derartige Fristen zum gewöhnlichen Geschäftsbetrieb der Kanzlei gehören; s. BVerwG NJW 1982, 2458, 2458.

44 BVerwG NJW 1995, 2122, 2123.

45 OVG Münster NVwZ-RR 2004, $221 \mathrm{f}$.

46 OVG Münster NJW 2014, 3117.

47 BGH NJW-RR 2012, 124; OVG Bautzen NVwZ-RR 2017, 755, 758.

48 BVerwG NJW 1995, 1443.

49 OVG Saarlouis NVwZ-RR 2015, 559. Zur Pflicht die Angestellten anzuweisen, nach Absenden eines Telefax die Richtigkeit des Adressaten und der Rufnummer zu prüfen, VGH München BayVBl. 2016, $719 \mathrm{f}$. Umfassend Kummer Wiedereinsetzung in den vorigen Stand, 2003, Rn. $388 \mathrm{ff}$.
$\S 85$ II ZPO. ${ }^{50}$ Im Übrigen ist der Behördenleiter zwar nicht als gesetzlicher Vertreter anzusehen. Eine Zurechnung seines Verschuldens nach dem Rechtsgedanken des § 51 II ZPO ist aber möglich. ${ }^{51}$ Das Verschulden anderer Bediensteter ist zurechenbar, wenn es Ausdruck eines Organisations- oder Überwachungsverschuldens des Behördenleiters ist ${ }^{52}$ oder der Bedienstete mit der selbstständigen Bearbeitung der Sache betraut war ${ }^{53}$.

Die Verschuldenszurechnung ist nur für die Dauer des Vertretungsverhältnisses möglich. Sobald das Mandatsverhältnis beendet ist, kommt eine Zurechnung nicht mehr in Betracht. Hierfür genügt es, dass die Beendigung des Mandatsverhältnisses im Innenverhältnis erfolgte. ${ }^{54}$ Eine Niederlegung des Mandats im Außenverhältnis ist dagegen nicht erforderlich. ${ }^{55}$

Lösung Fall 3 Ein Wiedereinsetzungsantrag des $K$ wäre zulässig aber unbegründet. Die Revisionsbegründungsfrist ist zwei Monate nach Zustellung des Urteils abgelaufen (\$ 139 III 1 VwGO), sodass die am Folgetag eingereichte Begründungsschrift verspätet war. Dem K fällt selbst kein Verschulden zur Last und auch eine Zurechnung des Verschuldens des $S$ ist nicht möglich, da dieser nicht von dem K bevollmächtigt wurde (§ 173 S. 1 VwGO i.V.m. § 85 ZPO). Allerdings hätte der R die Berechnung der Revisionsbegründungsfrist nicht dem $S$ überlassen dürfen, sondern eine eigene Berechnung vornehmen müssen. Dieses Verschulden ist dem K nach den genannten Vorschriften zuzurechnen.

\section{b) Kausalität}

Fall 4 K beantragt am 1. Juni 2017 eine straßenrechtliche Sondernutzungserlaubnis, um auf dem Gehweg vor seinem Restaurant eine Bestuhlung einzurichten. Am 7. August fährt er in den vierwöchigen Sommerurlaub. Am 8. August wird ihm ein abschlägiger Bescheid zugestellt. Am Tag seiner Rückkehr, dem 4. September, findet er den Bescheid in seinem Briefkasten und fragt Rechtsanwalt $R$, bis wann er Widerspruch gegen den Bescheid erheben muss und ob im Fall eines verspäteten Widerspruchs ein Wiedereinsetzungsantrag Aussicht auf Erfolg hätte.

Das unverschuldete Hindernis muss kausal für das Fristversäumnis sein. Liegen keine anderweitigen Anhaltspunkte vor, kann grundsätzlich von der Kausalität aus-

50 BVerwG NVwZ-RR 1996, 60, 61; Kuhla/Hüttenbrink Der Verwaltungsprozess, 3. Aufl. 2002, Rn. 406.

51 Sodan/Ziekow/Czybulka VwGO, 4. Aufl. 2014, § 60 Rn. 65. 52 VGH München BayVBl. 1973, 239, 240; Heiß BayVBl. 1984, 646, $649 \mathrm{f}$.

53 Sodan/Ziekow/Czybulka VwGO, 4. Aufl. 2014, § 60 Rn. 65. 54 BVerwG NVwZ 2000, 65, 65; BFH NVwZ 2002, 1401, 1402.

55 A.A. Kummer Wiedereinsetzung in den vorigen Stand, 2003, Rn. 397, der erst bei einer Mandatsniederlegung im Außenverhältnis die Verschuldenszurechnung enden lässt. 
gegangen werden. ${ }^{56}$ Nur in zwei Sonderkonstellationen ist sie problematisch:

Erstens ist bei einer Ursachenmehrheit eine wertende Betrachtung anzustellen. ${ }^{57}$ Entscheidend ist, ob das verschuldete Hindernis die Fristversäumnis prägt. ${ }^{58}$ Beruht das Fristversäumnis neben dem Verschulden des Antragstellers auch auf einem Verhalten eines Dritten, ist der Handlungsschwerpunkt maßgeblich. Hat der Antragsteller etwa ein Rechtsmittel bei dem Gericht eingelegt, dessen Entscheidung angefochten wird, ${ }^{59}$ kann er darauf vertrauen, dass es im ordentlichen Geschäftsgang an das höhere Gericht weiter geleitet wird. ${ }^{60}$ Legt er den Rechtsbehelf dagegen bei einer unzuständigen Stelle ein, kann er sich nicht darauf berufen, dass die Frist bei einer unverzüglichen Weiterleitung an die zuständige Stelle eingehalten worden wäre. ${ }^{61}$ Bei einer behördlichen oder gerichtlichen Mitschuld an dem Fristversäumnis ist der Betroffene allerdings mit besonderer Fairness zu behandeln. ${ }^{62}$

Fällt das Hindernis zweitens vor dem Ende der versäumten Frist weg, ist zu unterscheiden: Wenn zwischen dem Wegfall des Hindernisses und dem Fristablauf ein Zeitraum von mindestens zwei Wochen (§ 60 II 11 . Hs. VwGO) bzw. einen Monat ( 600 II 12 2. Hs. VwGO) liegt, kann keine Wiedereinsetzung verlangt werden. ${ }^{63}$ Die Wiedereinsetzungsfrist ist in diesem Fall nämlich nicht beeinträchtigt. Beträgt der Zeitraum dagegen weniger als zwei Wochen bzw. ein Monat, besteht Uneinigkeit. Teilweise wird davon ausgegangen, dass in diesen Fällen eine Wiedereinsetzung unbegründet ist. ${ }^{64}$ Der Antragsteller wäre nach dieser Ansicht verpflichtet, sofort nach Wegfall des unverschuldeten Hindernisses die versäumte Rechts-

56 In diese Richtung auch Hufen Verwaltungsprozessrecht, 10. Aufl. 2016, § 6 Rn. 34.

57 Gärditz/Krausnick VwGO, 2013, § 60 Rn. 62.

58 Stelkens/Bonk/Sachs/Kallerhoff/Stamm VwVfG, 9. Aufl. 2018, $\S 32 \mathrm{Rn} .13$.

59 Dies sehen §§ 124 a III 1, 139 I 1, 146 IV 2 VwGO vor.

60 BVerfGE 93, 99, $114 \mathrm{f}$.

61 BVerwGE 55, 61, 65f.; VGH Mannheim NVwZ-RR 1994, 474, 475; OVG Greifswald NVwZ 1999, 201; Kummer Wiedereinsetzung in den vorigen Stand, 2003, Rn. 300. A. A. Sodan/Ziekow/Czybulka VwGO, 4. Aufl. 2014, § 60 Rn. 78. Offenlassend für den Fall, dass das Rechtsmittel unmittelbar bei dem Rechtsmittelgericht eingelegt wurde, OVG Berlin-Brandenburg Beschl. v. 12.10.2016 - OVG 1 B 10.16, Rn. 17 (juris).

62 BVerfGE 110, 339, $342 \mathrm{ff}$.

63 Redeker/v. Oertzen VwGO, 16. Aufl. 2014, § 60 Rn. 14.

64 BayVerfGH NJW 1982, 2660, 2661; VGH Mannheim DÖV 1979, 303; Burkiczak/Dollinger/Schorkopf/Hammer BVerfGG, 2015, § 93 Rn. 55; Maunz/Schmidt-Bleibtreu/Bethge/Hömig BVerfGG, 52. EL. 2017, § 93 Rn. 61. Für das Zivilrecht auch Büttner Wiedereinsetzung, 1996, § 6 Rn. 6. handlung nachzuholen. Dies führt im Einzelfall aber zu einer unangemessenen Beeinträchtigung von Art. 19 IV 1, 103 I GG (s. oben, II.). Nach anderer Ansicht beginnt die Frist aus $\S 60$ II 1 VwGO mit Wegfall des Hindernisses erneut zu laufen. Der Antrag auf Wiedereinsetzung kann nach dieser Auffassung innerhalb von zwei Wochen bzw. einem Monat nach Wegfall des Hindernisses gestellt werden..$^{65} \S 60$ II 1 VwGO wird hiernach als »Mindestüberlegungsfrist « ausgelegt, was aber im Einzelfall zu schematisch erscheint. Bei einfach gelagerten Fällen kann dem Antragsteller nämlich zugemutet werden, einen Antrag auch vor Ablauf der Wiedereinsetzungsfrist von zwei Wochen bzw. einem Monat zu stellen. Am überzeugendsten ist daher die dritte, vermittelnde Ansicht, derzufolge eine Wiedereinsetzung innerhalb von zwei Wochen bzw. einem Monat erfolgen kann, wenn die verbleibende Frist gemessen an dem Schwierigkeitsgrad der Sache unangemessen kurz ist. ${ }^{66}$ Ist sie dagegen angemessen, muss die Rechtshandlung innerhalb der verbleibenden Restfrist nachgeholt werden. Der unbestimmte Rechtsbegriff der »Unangemessenheit« führt auch nicht zu Rechtsunsicherheit. Im Kern geht es hier nämlich um eine zeitspezifische Ausprägung des Verschuldensmaßstabs. Ein Verschulden liegt vor, wenn der Antragsteller nach Wegfall des Hindernisses nicht unverzüglich, d.h. ohne schuldhaftes Zögern (§ 121 I 1 BGB), den Antrag auf Wiedereinsetzung stellt. Die Schuldhaftigkeit eines Zögerns hängt wiederum von der Komplexität der Sache ab.

Lösung Fall 4 Die Widerspruchsfrist beträgt einen Monat (§ 70 I
VwGO), sodass K bis zum 8. September Widerspruch gegen den ihm
am 8. August zugestellten Bescheid erheben muss. Allerdings könn-
te K im Fall eines Fristversäumnisses ggf. Wiedereinsetzung in den
vorigen Stand mit der Begründung beantragen, dass er aufgrund
seines Urlaubs unverschuldet an der Einhaltung der Frist gehindert
war. Der Urlaub endete jedoch am 4. September und damit vor
Ablaufder Widerspruchsfrist.Die Widerspruchsbehörde könnteden
Antrag auf Wiedereinsetzung daher zurückweisen, weil das Hinder-
nis(Urlaub)nicht kausalfürdas Fristversäumniswar.Dem könnteK
allerdings entgegnen, dass dies eine unzulässige Verkürzung der
Zweiwochenfrist für die Wiedereinsetzung (§ 60 II 1. Hs. VwGO)
darstellt. Nach h.M. handelt es sich bei dieser jedoch nicht um eine
»Mindestüberlegungsfrist«. Vielmehr ist im Einzelfall zu prüfen, ob

65 VGH München BayVBl. 1980, 183; Kopp/Ramsauer VwVfG, 18. Aufl. 2017, § 32 Rn. 17; Kopp/Schenke VwGO, 23. Aufl. 2017, § 60 Rn. 7.

66 BVerwGE 88, 66, 69; NJW 1976, 74 f.; NVwZ-RR 1999, 472, 473; Beschl. v. 25.6.2013 - 10 B 10/13, Rn. 7 (juris); Sodan/Ziekow/Czybulka VwGO, 4. Aufl. 2014, § 60 Rn. 104; Mann/Sennekamp/Uechtritz/Mattes VwVfG, 2014, § 32 Rn. 23; Eyermann/Schmidt VwGO, 14. Aufl. 2014, § 60 Rn. 3. Der Sache nach auch Gräber/Stapperfend FGO, 8. Aufl. 2015, § 56 Rn. 34, der die zu kurze verbleibende Frist ihrerseits als ein die Wiedereinsetzung begründendes Hindernis ansieht. 
die für die Erhebung des Widerspruchs verbleibende Frist unter Beachtung der Komplexität der Sache angemessen ist. Zwischen Ks Rückkehr aus dem Urlaub und dem Ende der Widerspruchsfrist lagen vier Tage, was trotz der ggf. geringen Komplexität der Sachund Rechtslage eine unangemessen kurze Frist darstellt. Somit hätte ein Antrag auf Wiedereinsetzung in den vorigen Stand Aussicht auf Erfolg, wenn K seinen Widerspruch nach dem 8. September und vor dem18. September (zwei WochennachUrlaubsrückkehr) einhebt.

\section{c) Glaubhaftmachung}

Der Wiedereinsetzungsantrag ist - wenn er nicht offenkundig oder gerichtsbekannt ist ${ }^{67}$ - glaubhaft zu machen (§ 60 II 3 VwGO). ${ }^{68}$ Der Antragsteller muss mit überwiegender Wahrscheinlichkeit die Kausalität des unverschuldeten Hindernisses darlegen. Als Mittel der Glaubhaftmachung kommt neben den allgemeinen Beweismitteln insbesondere die eidesstattliche Versicherung in Betracht (§ 173 S. 1 VwGO i.V.m. § 294 ZPO). Das Vorliegen eines Fristversäumnisses - und damit die Statthaftigkeit des Antrags - ist durch das Gericht/die Behörde dagegen durch eine umfassende Beweisaufnahme zu klären. ${ }^{69}$

\section{Besonderheiten im Europarecht}

Die Wiedereinsetzung in den vorigen Stand hat im Europarecht keine mit dem deutschen Recht vergleichbare Dogmatisierung erfahren. Die hierzu vorhandene Rechtsprechung des EuGH ist noch überschaubar. ${ }^{70}$ Im Grundsatz sind die obigen Voraussetzungen zu der Zulässigkeit und Begründetheit der Wiedereinsetzung übertragbar. Besonderheiten ergeben sich aber aus dem nachfolgend Erläuterten.

67 BVerwG BayVBl. 1989, 122f.; OVG Münster NJW 1996, 2809; Kintz Öffentliches Recht im Assessorexamen, 8. Aufl. 2012, Rn. 286; Kopp/ Ramsauer VwVfG, 18. Aufl. 2017, § 32 Rn. 52.

68 §§ 32 II 2 VwVfG, 93 II 3 BVerfGG, 56 II 2 FGO, 67 II 2 SGG.

69 Schoch/Schneider/Bier/Steinbeiß-Winkelmann VwGO, 33. EL. 2017, § 60 Rn. 13.

70 EuGH C-25/65 u.a. - Simet, Slg. 1967, I-42 Rn. 56; C-284/82 Acciaierie Ferriere Busseni SpA, Slg. 1984, 557 Rn. 11; C-209/83 Ferriera Valsabbia SpA, Slg. 1984, 3089 Rn. 21; C-59/91 - Frankreich/ Kommission, Slg. 1992, I-525 Rn. 10; C-195/91 P-Bayer AG, Slg. 1994, I-5619 Rn. 32; C-239/97 - Irland/Kommission, Slg. 1998, I-2655 Rn. 7; C-325/03 P-Zuazaga Meabe, Slg. 2005, I-403 Rn. 25 ff.; C-242/07 - Belgien, Slg. 2007, I-9757 Rn. 16ff.; C-112/09 P-SGAE, Slg. 2010, I-351 Rn. 20 ff.; C-73/10 P-Weichert, Slg. 2010, I-11535 Rn. 41ff.; C-426/10 P-Bell \& Ross, Slg. 2011, I-8849 Rn. 47 ff.; C-478/11 P u.a. - Gbagbo, ECLI 2013:258 Rn. 68 ff.; EuG T-29/97 - Liberos, Slg. 2000 ÖD, I-A-43 Rn. 30; T-468/10 - Doherty, Slg. 2011, II-1497 Rn. $18 \mathrm{ff}$.

\section{Prozessrecht}

Die Wiedereinsetzung ist im Unionsrecht grundsätzlich ${ }^{71}$ bei allen prozessualen Fristen statthaft. ${ }^{72}$ Im Gegensatz zum deutschen Recht sieht Art. 45 II VerfO EuGH aber keine Frist für den Wiedereinsetzungsantrag vor. Teilweise wird zwar davon ausgegangen, dass der Antragsteller die Verfahrenshandlung unverzüglich nach Wegfall des Hindernisses nachholen muss. ${ }^{73}$ Ein solches ungeschriebenes Erfordernis ist aber nicht mit dem Wortlaut von Art. 45 II VerfO EuGH vereinbar und daher abzulehnen. Allenfalls aus dem Verbot des Rechtsmissbrauchs kann ein verspäteter Wiedereinsetzungsantrag in Einzelfällen zurückzuweisen sein.

Der Wiedereinsetzungsantrag ist nur begründet, wenn das Fristversäumnis auf Zufall oder höherer Gewalt beruht, ${ }^{74}$ was anzunehmen ist, wenn "ungewöhnliche, vom Willen des Betroffenen unabhängige Schwierigkeiten [vorliegen], die selbst bei Beachtung aller erforderlichen Sorgfalt unvermeidbar erscheinen. ${ }^{75} \mathrm{Im}$ Ergebnis läuft dies vergleichbar mit dem deutschen Recht - auf eine Verschuldensprüfung hinaus. ${ }^{76}$ Der Begriff der »höheren Gewalt« ist somit im EU-Recht weiter zu verstehen als im Rahmen des $\S 60$ III 2. Hs. VwGO. Er umfasst

»ein objektives und ein subjektives Merkmal [...], von denen ersteres sich auf ungewöhnliche, außerhalb der Sphäre des Wirtschaftsteilnehmers liegende Umstände bezieht und letzteres mit der Verpflichtung des Betroffenen zusammenhängt, sich gegen die Folgen ungewöhnlicher Ereignisse zu wappnen, indem er, ohne übermäßige Opfer zu bringen, geeignete Maßnahmen triff. ${ }^{17}$

71 Keine Anwendung findet Art. 45 II VerfO EuGH auf Art. 46 I, 44 III EuGH-Satzung; s. v. d. Groeben/Schwarze/Hatje/Dittert Europäisches Unionsrecht, Bd. 4, 7. Aufl. 2015, Art. 45 EuGH-Satzung Rn. 5.

72 Art. 258 II, 263 VI 1. Hs., 265 II 2, 269 II AEUV; Art. 23 II, 56 I 2. Hs. EuGH-Satzung; Art. $41 \S 2,115 \S 1$ VerfO EuG.

73 v. d. Groeben/Schwarze/Hatje/Dittert Europäisches Unionsrecht, Bd. 4, 7. Aufl. 2015, Art. 45 EuGH-Satzung Rn. 13.

74 Hierzu Rengeling/Middeke/Gellermann/Neumann Handbuch des Rechtsschutzes in der EU, 3. Aufl. 2014, § 30 Rn. 10. Eine trennscharfe Unterscheidung dieser Kategorien nimmt der EuGH nicht vor; vgl. v. d. Groeben/Schwarze/Hatje/Dittert Europäisches Unionsrecht, Bd. 4, 7. Aufl. 2015, Art. 45 EuGH-Satzung Rn. 6.

75 EuGH C-284/82 - Acciaierie Ferriere Busseni SpA, Slg. 1984, 557 Rn. 11; 209/83 - Ferriera Valsabbia SpA, Slg. 1984, 3089 Rn. 21.

76 So auch Pechstein EU-Prozessrecht, 4. Aufl. 2011, Rn. 185. Für eine restriktive Auslegung aber Pechstein/Nowak/Häde/Pechstein/Görlitz Frankfurter Kommentar zu EUV, GRC und AEUV, 2017, Art. 263 AEUV Rn. 165.

77 EuGH C-195/91 P-Bayer AG, Slg. 1994, I-5619 Rn. 32; C-325/03 P-Zuazaga Meabe, Slg. 2005, I-403 Rn. 25; C-242/07 - Belgien, 
Als besonderen Wiedereinsetzungsgrund erkennt der EuGH zudem den »entschuldbaren Irrtum« an, der in Fällen vorliegt,

»in denen das betroffene Organ ein Verhalten an den Tag gelegt hat, das [...] geeignet gewesen ist, bei einem gutgläubigen Rechtsbürger, der alle Sorgfalt aufwendet, die von einem Wirtschaftsteilnehmer mit normalem Kenntnisstand verlangt werden kann, eine verständliche Verwirrung hervorzurufen. "78 $^{7}$

\section{Verwaltungsverfahrensrecht}

Im Kontext des EU-Verwaltungsverfahrensrechts ist $\mathrm{zu}$ differenzieren: Wird das anwendbare Unionsrecht durch die Mitgliedstaaten vollzogen (indirekter Vollzug), kann die nationale Behörde bei einem Versäumnis einer materiell-rechtlichen Frist nach den Vorschriften des nationalen Rechts (in Deutschland § 32 [L]VwVfG) Wiedereinsetzung in den vorigen Stand gewähren. Bei der Auslegung und Anwendung des nationalen Rechts sind aber wegen Art. 4 III EUV (Prinzip der loyalen Zusammenarbeit) die Wirksamkeit des Unionsrechts zu gewährleisten (Effektivitätsprinzip) und eine diskriminierende Behandlung gegenüber vergleichbaren innerstaatlichen Sachverhalten $\mathrm{zu}$ vermeiden (Äquivalenzprinzip). ${ }^{79}$ Sind dagegen die Unionsbehörden ausnahmsweise selbst für den Vollzug des Unionsrechts zuständig (direkter Vollzug), können sie Wiedereinsetzung gewähren, wenn die Fristversäumnis auf einem entschuldbaren Irrtum beruht. ${ }^{80}$
Slg. 2007, I-9757 Rn. 17; C-426/10 P-Bell \& Ross, Slg. 2011, I-8849 Rn. 48.

78 EuGH C-73/10 P-Weichert, Slg. 2010, I-11535 Rn. 42; C-242/07 Belgien, Slg. 2007, I-9757 Rn. 29; C-112/09 P-SGAE, Slg. 2010, I-351 Rn. 20; EuG T-514/93 - Cobrecaf, Slg. 1995, II-621 Rn. 40; T-29/97 Liberos, Slg. 2000 ÖD, I-A-43 Rn. 30.

79 EuGH C-285/93 - Dominikanerinnen-Kloster-Altenhohenau, Slg. 1995, I-4069 Rn. 28ff.; Kopp/Ramsauer VwVfG, 18. Aufl. 2017, $\S 32$ Rn. 3 a.

80 EuGH C-84/08 P-Pitsiorlas/Rat und EZB, Slg. 2008 I-104, Rn. $20 \mathrm{ff}$.

\section{Entscheidung über die Wiedereinsetzung und Rechtsschutz}

\section{Entscheidung}

Über den Wiedereinsetzungsantrag ist in der Form zu entscheiden, in der über die versäumte Rechtshandlung zu entscheiden wäre. ${ }^{81}$ Das Gericht trifft seine Entscheidung somit im Rahmen des Urteils oder Beschlussesüber die Hauptsache ${ }^{82}$, die Behörde durch Verwaltungsakt ${ }^{83}$.

Wird dem Wiedereinsetzungsantrag stattgegeben, gilt die Handlung als rechtzeitig bewirkt. ${ }^{84}$ Das Verfahren wird in den Stand versetzt, in dem es sich vor der Fristversäumnis befand..$^{85}$ Ist der verfahrensgegenständliche Verwaltungsakt oder die gerichtliche Entscheidung über diesen bereits bestands- bzw. rechtskräftig geworden, entfällt die Bestands- bzw. Rechtskraft mit Gewährung der Wiedereinsetzung. ${ }^{86}$ Einer gesonderten Aufhebung bedarf es nicht.

\section{Rechtsschutz}

Fall 5 Dem B wird eine Baugenehmigung erteilt, mit der er sein Grundstück bis an die Grenze des Grundstücks seines Nachbarn N bebauen möchte. $N$ legt verspätet Widerspruch gegen die Baugenehmigung ein und beantragt Wiedereinsetzung in den vorigen Stand. Die Widerspruchsbehörde gibt dem Wiedereinsetzungsantrag des $N$ statt und hebt die dem B erteilte Baugenehmigung auf. B ist der Auffassung, der verspätete Widerspruch beruhe auf einem Verschulden des N. Welchen Rechtsbehelf kann B erheben? Worauf ist er zu richten?

Fall 6 Die Widerspruchsbehörde verwirft den in Fall 4 am 11. September eingelegten Widerspruch von K mit der Begründung, dass

81 Eyermann/Schmidt VwGO, 14. Aufl. 2014, § 60 Rn. 30.

82 Teilweise wird auch ein gesonderter Vorab-Beschluss über das Wiedereinsetzungsgesuch als zulässig erachtet (so Kuhla/Hüttenbrink Der Verwaltungsprozess, 3. Aufl. 2002, Rn. 412; Redeker/v. Oertzen VwGO, 16. Aufl. 2014, § 60 Rn. 20; Seubert BayVBl. 1963, 208, $208 \mathrm{ff}$.), was wegen des Wortlauts von $\S 60$ IV VwGO (»entscheidet« nicht »beschließt«) nach a. A. zurückgewiesen wird (OVG Berlin-Brandenburg NVwZ-RR 1990, 388f.; Kopp/Schenke VwGO, 23. Aufl. 2017, § 60 Rn. 37).

83 Hierbei handelt es sich um einen rechtsgestaltenden Verwaltungsakt; s. Buri DÖV 1963, 498, 499; Weides Verwaltungsverfahren und Widerspruchsverfahren, 3. Aufl. 1993, 266.

84 Zöller/Greger ZPO, 32. Aufl. 2018, § 233 Rn. 1.

85 Schoch/Schneider/Bier/Steinbeiß-Winkelmann VwGO, 33. EL. 2017, § 60 Rn. 11.

86 Posser/Wolff/Brink BeckOK VwGO, 43. Ed. 2017, § 60 Rn. 29; Kopp/RamsauerVwVfG, 18. Aufl. 2017, § 32 Rn. 62. 
er verspätet eingegangen und damit unzulässig sei. Den Antrag auf Wiedereinsetzung weist sie als unbegründet zurück. Hiergegen möchte $\mathrm{K}$ vorgehen. Welche Rechtsbehelfe stehen ihm zur Verfügung?

Im Einzelfall kann fraglich sein, welche Rechtsbehelfe einem Betroffenen gegen eine (nicht) gewährte Wiedereinsetzung zustehen. Diesbezüglich ist zwischen der Wiedereinsetzung im Verwaltungs-, Widerspruchs- und Gerichtsverfahren zu unterscheiden.

\section{a) Verwaltungsgerichtliches Verfahren}

Im verwaltungsgerichtlichen Verfahren gilt Folgendes: Wird die Wiedereinsetzung gewährt, ist die gerichtliche Entscheidung unanfechtbar ( $\$ 60 \mathrm{~V}$ VwGO). ${ }^{87}$ Wird die Wiedereinsetzung dagegen versagt, kann sich der Antragsteller mit den gegen die Hauptsacheentscheidung statthaften Rechtsbehelfen zur Wehr setzen. ${ }^{88}$ Fraglich ist dann, ob das Ausgangs- oder das Rechtsmittelgericht für die Entscheidung über die Wiedereinsetzung zuständig ist. Teilweise wird davon ausgegangen, das Rechtsmittelgericht entscheide selbst über die Wiedereinsetzung. ${ }^{89}$ Nach der zutreffenden Meinung kann das Rechtsmittelgericht aber nicht alleine über die Rechtmäßigkeit der Ablehnung des Wiedereinsetzungsgesuchs entscheiden. Wenn es die Ablehnung für rechtswidrig hält, muss es die Sache an das Ausgangsgericht zurückverweisen. ${ }^{90}$ Würde das Rechtsmittelgericht nämlich selbstständig über die Wiedereinsetzung entscheiden, müsste es zugleich in der Sache entscheiden, wodurch dem Rechtsmittelführer eine ihm ansonsten zustehende Instanz verloren ginge.

\section{b) Widerspruchsverfahren}

Der für das Widerspruchsverfahren maßgebliche $\S 70$ II VwGO enthält eine auf $\S 60$ I-IV VwGO beschränkte Rechtsgrundverweisung. $\S 60 \mathrm{~V}$ VwGO ist im Wider-

87 S. auch $\S \S 56$ V FGO, 67 IV 2 SGG.

88 Kuhla/Hüttenbrink Der Verwaltungsprozess, 3. Aufl. 2002, Rn. 413.

89 BFH NJW 1983, 1872; Schoch/Schneider/Bier/Steinbeiß-Winkelmann VwGO, 33. EL. 2017, § 60 Rn. 71; Kopp/Schenke VwGO, 23. Aufl. 2017, § 60 Rn. 35.

90 BGH NJW 1982, 887 f.; Redeker/v. Oertzen VwGO, 16. Aufl. 2014, $\S 60$ Rn. 21a; Sodan/Ziekow/Czybulka VwGO, 4. Aufl. 2014, § 60 Rn. 138; Gärditz/Krausnick VwGO, 2013, § 60 Rn. 83. Für den Fall, dass der Wiedereinsetzungsantrag erst in der Berufungsinstanz gestellt wird, s. auch BVerwG NVwZ 1985, 484, 485. spruchsverfahren nicht anwendbar, sodass grundsätzlich sowohl gegen die Gewährung als auch gegen die Versagung der Wiedereinsetzung vorgegangen werden kann. Beim Rechtsschutz gegen die Gewährung der Wiedereinsetzung geht es regelmäßig um Drittbeteiligungsfälle. Entweder hat ein Dritter Widerspruch gegen einen Verwaltungsakt eingelegt, der für den Adressaten begünstigend, für den Dritten dagegen belastend ist (Konstellation 1), oder der Adressat hat gegen einen ihn belastenden Verwaltungsakt Widerspruch eingelegt, welcher einen Dritten begünstigt (Konstellation 2). War der Widerspruch verspätet und hat die Widerspruchsbehörde Wiedereinsetzung gewährt, kann der durch den Verwaltungsakt begünstigte Adressat (Konstellation 1) bzw. der Drittbegünstigte (Konstellation 2) Anfechtungsklage gegen den Widerspruchsbescheid erheben. Das Gericht entscheidet dann über die Rechtmäßigkeit der Wiedereinsetzung durch die Widerspruchsbehörde. Es prüft die Rechtmäßigkeit einer durch die Widerspruchsbehörde gewährten Wiedereinsetzung aber nur, wenn der Kläger deren Rechtswidrigkeit gerügt hat. ${ }^{91}$ Eine isolierteAnfechtung der Wiedereinsetzung ist dagegen nicht statthaft (§ $44 \mathrm{a}$ VwGO). ${ }^{92}$

\begin{abstract}
Lösung Fall 5 B könnte Anfechtungsklage erheben, die auf die Aufhebung des Widerspruchsbescheids zu richten wäre (\$§ $68 \mathrm{I}$, 79 I Nr. 2 VwGO). Eine isolierte Anfechtung der zugunsten des $N$ erfolgten Wiedereinsetzung scheitert an $\S 44$ a VwGO. Das Gericht prüft die Zulässigkeit und Begründetheit des Widerspruchs des N. Im Rahmen der Zulässigkeit überprüft das Gericht auch den Wiedereinsetzungsantrag. Kommt es zu dem Ergebnis, dass dem $N$ Wiedereinsetzung zu gewähren war, prüft das Gericht im Rahmen der Begründetheit des Widerspruchs die Rechtmäßigkeit der dem B erteilten Baugenehmigung.
\end{abstract}

Die Frage nach dem statthaften Rechtsbehelf stellt sich auch, wenn ein Antrag auf Wiedereinsetzung durch die Widerspruchsbehörde abgelehnt wurde. In diesem Fall kann der Widerspruchsführer Verpflichtungsklage erheben. ${ }^{93}$ Das Verwaltungsgericht prüft dann, ob die Wieder-

91 VGH Kassel NVwZ-RR 1993， 432， 434; VGH Mannheim NVwZ-RR 2002, 6, 6; Eyermann/Rennert VwGO, 14. Aufl. 2014, § 70 Rn. 13; Ganter VBlBW 1984, 402, 402f. A. A. Kopp/Schenke VwGO, 23. Aufl. 2017, $\S 70$ Rn. 13, der eine Pflicht zur Prüfung von Amts wegen annimmt.

92 Hufen Verwaltungsprozessrecht, 10. Aufl. 2016, § 6 Rn. 36. Zur Rechtslage vor Schaffung des § 44a VwGO s. Buri DÖV 1963, 498, 499.

93 Allerdings muss der Kläger im Rahmen des Rechtsschutzbedürfnisses darlegen, dass er ein besonderes Interesse an einer erneuten behördlichen Sachentscheidung hat; vgl. Ganter VBlBW 1984, 402, 404f.; Eyermann/Rennert VwGO, 14. Aufl. 2014, § 70 Rn. 14f. Insgesamt gegen die Zulässigkeit einer Verpflichtungsklage Kopp/Schenke VwGO, 23. Aufl. 2017, § 70 Rn. 15. 
einsetzung zu Unrecht abgelehnt wurde. Ist dies der Fall, verpflichtet es die Widerspruchsbehörde, dem Widerspruchsführer Wiedereinsetzung zu gewähren und neu über die Sache zu entscheiden. Daneben kann der Widerspruchsführer nach h.M. auch eine Anfechtungsklage gegen den Widerspruchsbescheid erheben; ${ }^{94}$ das Gericht gewährt dann selbstständig Wiedereinsetzung und entscheidet in der Sache. ${ }^{95}$ Teilweise wird die Zulässigkeit der Anfechtungsklage mit dem Argument bestritten, die Sachentscheidung des Gerichts greife in einer dem Gewaltenteilungsgrundsatz widersprechenden Weise in die behördliche Zuständigkeit ein. ${ }^{96}$ Ein Verstoß gegen den Gewaltenteilungsgrundsatz liegt jedoch nicht vor, da dem Gericht gerade die Aufgabe zukommt, die Entscheidung der Behörde in der Sache zu überprüfen. Zudem wäre es unpraktikabel, wenn der Antragsteller zunächst eine Verpflichtungsklage auf Gewährung der Wiedereinsetzung und anschließend eine gegen den Widerspruchsbescheid gerichtete Anfechtungsklage erheben müsste.

\begin{abstract}
Lösung Fall 6 K könnte nach der h.M. Anfechtungsklage gegen den Ablehnungsbescheid in Gestalt des Widerspruchsbescheids erheben (\$§ 68 I, 79 I Nr. 1 VwGO). Das Verwaltungsgericht prüft dann, ob die Widerspruchsbehörde den Widerspruch zu Recht als unzulässig verworfen und dem Wiedereinsetzungsantrag nicht stattgegeben hat. Kommt es zu dem Ergebnis, dass dem Wiedereinsetzungsantrag stattzugeben war, trifft das Gericht selbst eine Entscheidung in der Sache. Es verweist die Sache nicht an die Widerspruchsbehörde zurück. Das Verwaltungsgericht würde dann selbst über den Antrag des K auf Erteilung der Sondernutzungserlaubnis entscheiden. Daneben könnte $K$ eine Verpflichtungsklage erheben, die auf die Gewährung von Wiedereinsetzung durch die Widerspruchsbehörde zu richten wäre. Im Erfolgsfall würde das Verwaltungsgericht die Widerspruchsbehörde verpflichten, dem $K$ Wiedereinsetzung zu gewähren und über seinen Sondernutzungsantrag zu entscheiden. Da der K sein Ziel durch die Anfechtungsklage aber einfacher und ebenso effektiv erreichen
\end{abstract}

94 BVerwG NJW 1983, 1923, 1923; Hufen Verwaltungsprozessrecht, 10. Aufl. 2016, § 6 Rn. 36; Sodan/Ziekow/Geis VwGO, 4. Aufl. 2014, $\S 70$ Rn. 56 ff.; Klemm NVwZ 1989, 102, 109; Pietzner/Ronellenfitsch Das Assessorexamen im Öffentlichen Recht, 13. Aufl. 2014, Rn. 1152; Meyer-Ladewig/Keller/Leitherer/Keller SGG， 12. Aufl. 2017, §67 Rn. 13 a.

95 Die Zuständigkeit des Gerichts für die Gewährung der Wiedereinsetzung ist heute weitgehend anerkannt; vgl. nur BVerwG NJW 1983, 1923, 1923; BFHE 147, 407, 409; VGH Mannheim NJW 1970, 347 f.; DÖV 1981, 228f.; Eyermann/Rennert VwGO, 14. Aufl. 2014, §70 Rn. 14. A. A. Schmidt DÖV 1981, 229, 230; Wallerath DÖV 1973, 653, 657, denen zufolge das Gericht die Behörde zur Gewährung der Wiedereinsetzung verpflichtet, ohne selbst in der Sache zu entscheiden. 96 OVG Lüneburg DVBl. 1963, 335; VGH Mannheim NJW 1973, 727; NVwZ 1982, 316, 316f.; Buri DÖV 1963, 498, 499 ff.; Schmidt DÖV 1981, 229, 230; Weides Verwaltungsverfahren und Widerspruchsverfahren, 3. Aufl. 1993, 268. kann, müsste er ein besonderes Interesse an einer Sachentscheidung durch die Behörde darlegen, was vorliegend nicht ersichtlich ist. Daher wäre eine Verpflichtungsklage mangels Rechtsschutzbedürfnisses unzulässig.

\section{c) Verwaltungsverfahren}

Auch im Verwaltungsverfahren ist sowohl die Gewährung als auch die Versagung der Wiedereinsetzung anfechtbar. Eine mit $\S 60 \mathrm{~V}$ VwGO vergleichbare Vorschrift existiert nicht. Der Betroffene kann Widerspruch und - falls diesem nicht abgeholfen wird - Klage erheben. Gegenstand des Widerspruchs/der Klage ist aber nicht die Gewährung oder Versagung der Wiedereinsetzung selbst, ${ }^{97}$ da sie als unselbstständige Verfahrenshandlung i.S.d. § 44a VwGO anzusehen ist. Vielmehr sind Widerspruch und Klage auf die Sachentscheidung zu richten. Die Widerspruchsbehörde/Das Gericht prüft dann die Rechtmäßigkeit der Wiedereinsetzung inzident. ${ }^{98}$

\section{Zusammenfassung}

Hat eine Person eine Frist zur Vornahme einer öffentlichrechtlichen Rechtshandlung unverschuldet versäumt, kann ihr Wiedereinsetzung in den vorigen Stand gewährt werden. Die versäumte Rechtshandlung gilt dann als rechtzeitig bewirkt. Dieser allgemeine Rechtsgrundsatz findet Anwendung bei der Versäumnis einer Frist zur Einlegung eines Rechtsbehelfs im Verwaltungs- ( $\$ 60$ VwGO), Verfassungs- (§ 93 II BVerfGG) und europäischen Prozessrecht (Art. 45 II VerfO EuGH). Auch gegenüber einer Verwaltungsbehörde (§ 32 [L]VwVfG) sowie im Widerspruchsverfahren (§ 70 II i.V.m. $\S 60$ VwGO) kann ein Antragsteller sich auf dieses Institut berufen.

In Prüfungsarbeiten taucht die Wiedereinsetzung häufig in Kombination mit Problemen der Fristberechnung und des Zustellungsrechts auf. Im Rahmen der Zulässigkeit eines Rechtsbehelfs ist dann zunächst zu prüfen, ob der Rechtsbehelf verfristet war. Nur wenn dies der Fall ist, ist inzident (ggf. ausführlich!) die Zulässigkeit und Begründetheit eines Wiedereinsetzungsantrags zu prüfen. Der Schwerpunkt der Prüfung liegt dabei regelmäßig auf der Frage, ob der Antragsteller ohne Verschulden an der Ein-

97 So aber VGH Mannheim NJW 1972, 461; Kopp/Ramsauer VwVfG, 18. Aufl. 2017, § 32 Rn. 63.

98 Stelkens/Bonk/Sachs/Kallerhoff/Stamm VwVfG, 9. Aufl. 2018, $\S 32$ Rn. 45; Klemm NVwZ 1989, 102, 109; Mann/Sennekamp/Uechtritz/Mattes VwVfG, 2014, § 32 Rn. $83 \mathrm{f}$. 
haltung der Frist gehindert war, wobei besonderes Augenmerk auf die Zurechnung von Anwaltsverschulden zu legen ist.

Eine gelungene Prüfungsleistung zeichnet sich dabei nicht durch Detailkenntnis der jedenfalls für den Prü- fungsteilnehmer nicht $\mathrm{zu}$ überschauenden Rechtsprechung aus. Entscheidend sind vielmehr ein klar strukturierter Aufbau sowie ein Verständnis der Grundzüge des Wiedereinsetzungsrechts unter Berücksichtigung seines verfassungsrechtlichen Hintergrunds. 